\title{
Unsteady flow around a two-dimensional section of a vertical axis turbine for tidal stream energy conversion
}

\author{
Hyun Ju Jung ${ }^{1}$, Ju Hyun Lee ${ }^{2}$, Shin Hyung Rhee ${ }^{3}$, Museok Song ${ }^{4}$ and Beom-Soo Hyun ${ }^{5}$ \\ ${ }^{I}$ Next E\&S, Seoul, Korea \\ ${ }^{2}$ Dept. of Naval Architecture \& Ocean Engineering, Seoul National University, Seoul, Korea \\ ${ }^{3}$ Research Institute of Marine Systems Engineering, Seoul National University, Seoul, Korea \\ ${ }^{4}$ Dept. of Naval Architecture \& Ocean Engineering, Hongik University, Jochiwon, Chung-Nam, Korea \\ ${ }^{5}$ Div. of Naval Architecture \& Ocean Systems Engineering, Korea Maritime University, Busan, Korea
}

\begin{abstract}
The two-dimensional unsteady flow around a vertical axis turbine for tidal stream energy conversion was investigated using a computational fluid dynamics tool solving the Reynolds-Averaged Navier-Stokes equations. The geometry of the turbine blade section was NACA653-018 airfoil. The computational analysis was done at several different angles of attack and the results were compared with the corresponding experimental data for validation and calibration. Simulations were then carried out for the two-dimensional cross section of a vertical axis turbine. The simulation results demonstrated the usefulness of the method for the typical unsteady flows around vertical axis turbines. The optimum turbine efficiency was achieved for carefully selected combinations of the number of blades and tip speed ratios.
\end{abstract}

KEY WORDS: Tidal Stream Energy Conversion; Vertical Axis Turbine; Computational Fluid Dynamics.

\section{INTRODUCTION}

A typical tidal stream energy power generator consists of the energy conversion equipment for the kinetic energy of the tidal stream to run the electric generator and the support structures. Due to the intense push for the utilization of renewable energy recently, many different methods for generating electric energy using various forms of ocean energy are being investigated and realized. Among them, the tidal stream is considered as one of the most efficient and inexpensive renewable energy sources from the ocean. At the core of the research activities for the tidal stream energy conversion are the turbines that utilize the lift and drag forces acting on the blades. Largely the turbines are categorized into the horizontal axis turbine (HAT, Fig. 1) and vertical axis turbine (VAT, Fig. 2), depending on the axis alignment.

The HAT is the reverse concept of a marine propeller. The rotation is obtained by the lift force on the turbine blades, and the axis is parallel to the flow direction. In general, they are similar to wind turbines, and the simple mechanism is a big advantage. However, the turbine axis should be parallel to the stream direction to maximize the efficiency, and, for fixed pitch blades, the rotation direction needs to be reversed

Corresponding author: Shin Hyung Rhee

e-mail:shr@snu.ac.kr when the flow direction is reversed. To optimize the efficiency of the HAT, the turbine diameter $(D)$, numbers of blades, pitch variation in the radial direction, and the chord length (or expanded area ratio) should be determined carefully with the constraints such as the tidal stream characteristics, space, design power, and cavitation.

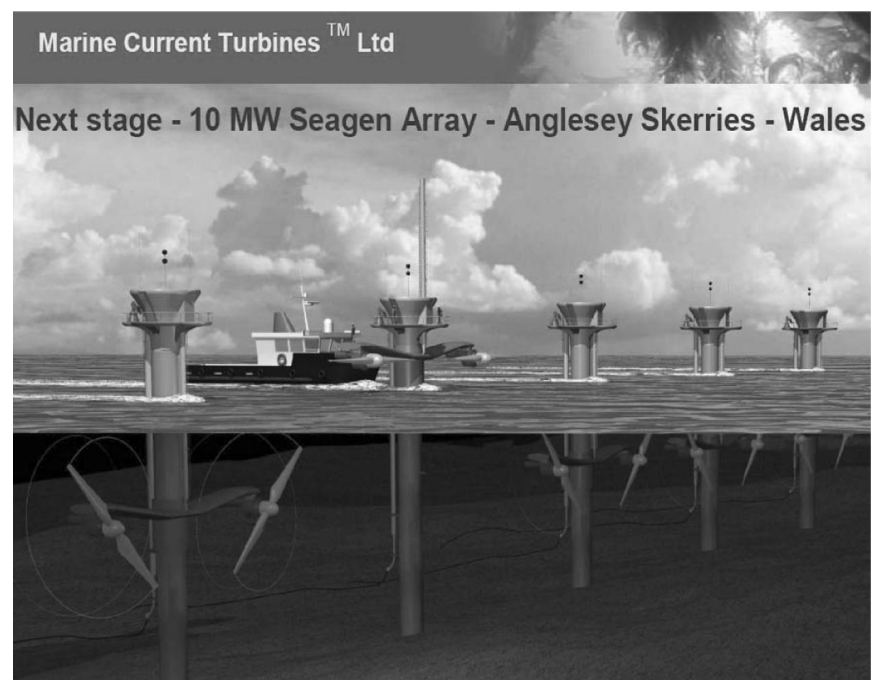

Fig. 1 Schematic picture of typical HAT (MCT). 


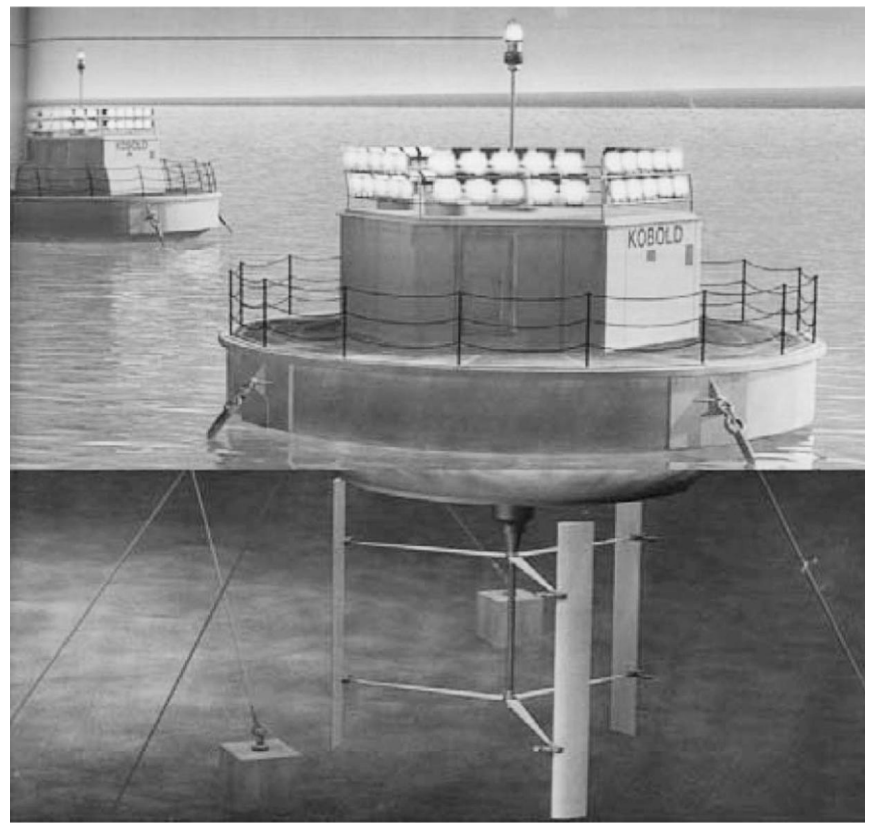

Fig. 2 Schematic picture of typical VAT (Kobold).

On the other hand, the VAT's axis of rotation is perpendicular to the flow direction (see Fig. 3). Compared to the HAT, its cut-in speed is high and efficiency is low. However, the rotation direction is independent of the flow direction and it is easy to set up the generator above the water surface. As for the HAT, the efficiency of the VAT can be optimized with properly determined turbine diameter, number of blades, pitch angle and blade chord length.

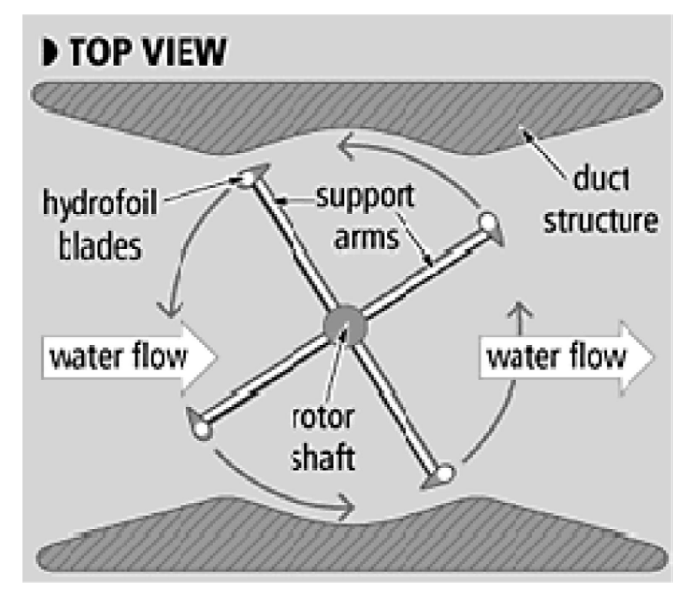

Fig. 3 A typical VAT (Darrieus turbine) arrangement (Blue Energy).

Most of the research activities on the turbine performance analysis have been found for wind turbines, using the blade element momentum theory (BEMT), which was introduced by Glauert (1963). Recently, due to the high interest in the renewable energy technology and great advancement of computer, computational fluid dynamics (CFD) codes solving the Reynods-Averaged Navier-Stokes (RANS) equations are applied in many different ways. The numerical analysis of the flow fields around wind turbines were performed mainly by nationally funded laboratories such as RiSo and the Danish Technical University in Denmark, the German Aerospace Center (DLR) in Germany, the Aeronautical Research Institute (FFA) in Sweden and NASA in USA. Especially, the National Renewable Energy Laboratory (NREL) test performed at NASA and NREL/NWTC aerodynamic blind comparison test (Sorensen et al., 2002) showed good application examples of CFD.

On the other hand, the computational research on the tidal stream turbines is difficult to find in the literature. Therefore, the ultimate goal of this study is the development of an analysis method for the VAT design. The present paper is the first report of the study. First, the flow around the NACA65 $3^{-}$ 018 airfoil, which is often employed for wind turbines, was simulated and analyzed for different angles of attack. The results were validated against the experimental data to verify the performance of the code. Then the computational analysis of the two-dimensional (2D) unsteady flow around the VAT was performed. The commercial CFD software, FLUENT v6.3, was used for the analysis. Different tip-speed ratios (TSR) of the turbine and different number of blades were also studied, and it was found that the turbine efficiency can be optimized with a proper combination of these parameters.

The present paper is organized as follows. The model problem is presented next, and followed by the computational method used. Then the computational results are presented and discussed. Finally, concluding remarks are made with a summary.

\section{MODEL PROBLEM}

The symmetric laminar airfoil $N A C A 65_{3}-018$ (Fig. 4) was selected, which is typical blade section geometry for wind turbines.

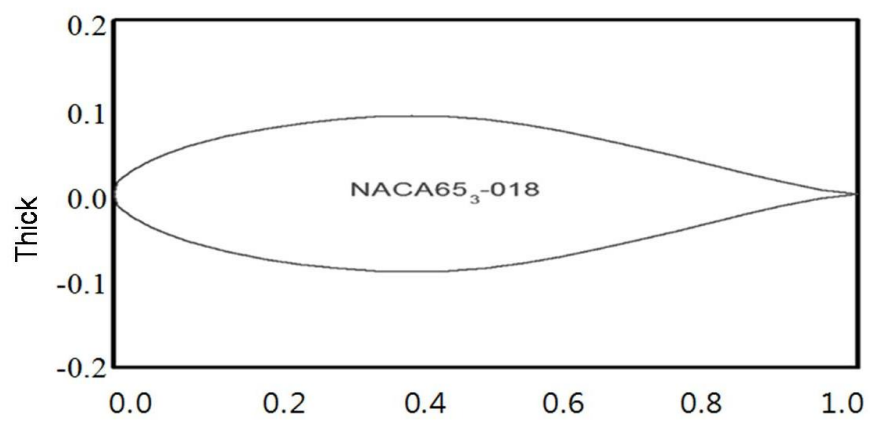

Fig. 4 NACA65 ${ }_{3}-018$ blade section.

The operating speed, expressed by TSR, was set between 2 and 3.5. Note that TSR is defined as $T S R=R \Omega / V$, where $R$ is 
the turbine radius, $\Omega$ is the angular velocity, and $V$ is the speed of the stream. The corresponding chord-based Reynolds number $(R e)$ was 4 million to 7 million with the chord length of $1 \mathrm{~m}$. In this $R e$ range, the laminar-to-turbulent transition did not takes place on the of blade surface, so no special treatment was needed. The flow condition for the analysis is shown in Table 1 . The turbine radius was set to 4 $m$, which is considered to be a better size to minimize the interference between blades.

Table 1 Flow condition.

\begin{tabular}{cccc}
\hline TSR & $\begin{array}{c}\text { Current } \\
\text { Speed }(\mathrm{m} / \mathrm{s})\end{array}$ & $\begin{array}{c}\text { Turbine's } \\
\text { Angular } \\
\text { speed }(\text { rad } / \mathrm{s})\end{array}$ & $\begin{array}{c}\text { Re } \\
\text { (Million) }\end{array}$ \\
\hline 2 & 2 & 1.00 & 3.98 \\
\hline 2.5 & 2 & 1.25 & 4.98 \\
\hline 3 & 2 & 1.50 & 7.97 \\
\hline 3.5 & 2 & 1.75 & 6.97 \\
\hline
\end{tabular}

The domain size was set large enough, based on other external flow analysis results. As shown in Fig. 5, there was the rotational sub-domain surrounding the blades and stationary sub-domains in the remaining region. The inlet width was set to $6 D$, while the outlet width was larger than that of the inlet, i.e., $10 D$. The inlet was located $3 D$ upstream and $12 D$ downstream.

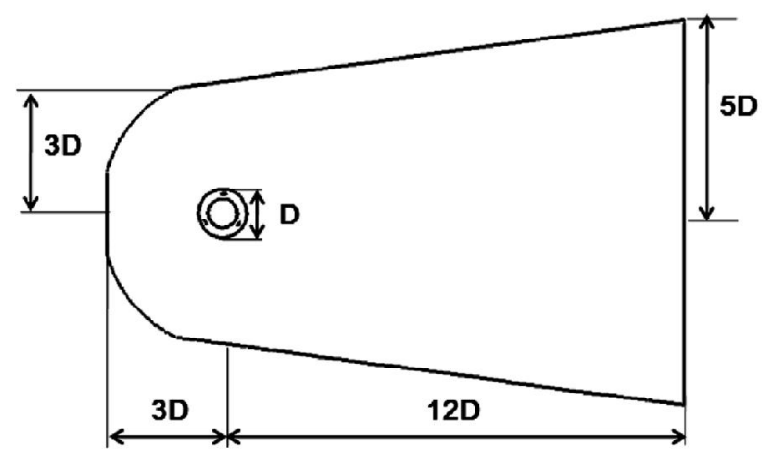

Fig. 5 Computational domain.

\section{COMPUTATIONAL METHOD}

The flow filed was simulated using the commercial CFD code, FLUENT v6.3. The same code has been used successfully for similar problems, such as Rhee and Kim (2008), Rhee (2009), and Ockfen and Matveev (2009). The RANS equations were solved using the cell-centered finite volume method, and the sliding mesh method was used to rotate the sub-domain for the turbine blades. The SIMPLE algorithm introduced by Patankar and Spalding (1972) was used for the velocity-pressure coupling. The QUICK scheme was employed to discretize the convection terms, while the $2^{\text {nd }}$ order accurate central difference scheme was used for the discretization of diffusion terms. Time integration was done implicitly, and the multi-grid method was used to accelerate the convergence. The k- $\omega$ SST model (Menter, 1993) was adopted for turbulence closure. Once the steady flow solution without rotating the turbine blades was obtained, the unsteady flow computation was commenced by turning on the blade rotation.

The sub-domain for the rotating turbine blades was shaped like a donut. The non-matching interface was defined between the rotating sub-domain and stationary outer subdomain, and the simple linear interpolation was used for the transition of the solution through the interface. Fig. 6 displays the location of the boundaries. The inlet was defined as a velocity inlet, which has constant inflow velocity, while the outlet was set as a pressure outlet, i.e., the pressure was kept constant. The velocity at the outlet was determined by the extrapolation from inside. The no-slip condition was applied on the turbine blades, i.e., set the relative velocity of blades to zero.

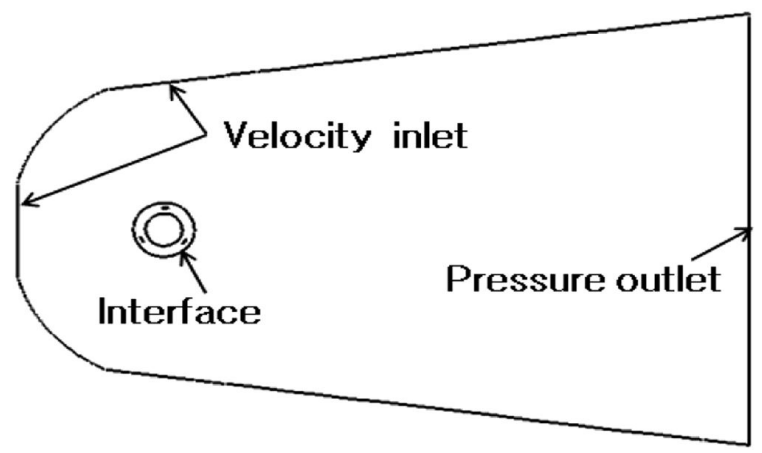

Fig. 6 Boundary conditions.

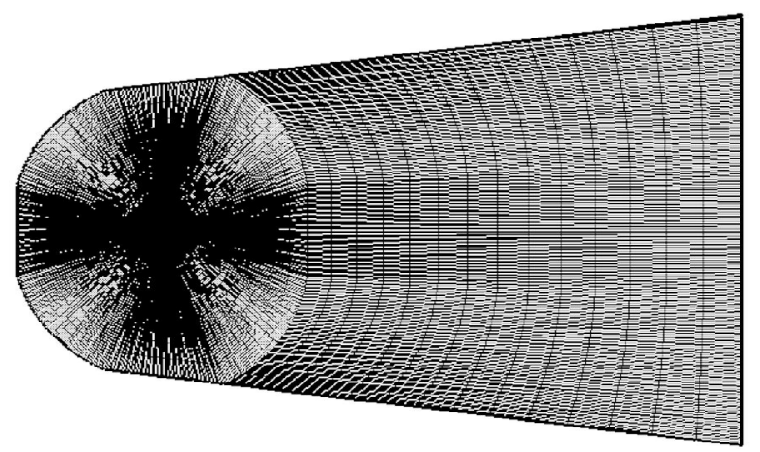

Fig. 7 Overall view of the mesh.

The mesh was generated with quadrilateral cells, except for the center of the rotation, where triangular cells were used. 
There were four sub-domains in the computational domain with 194,299 and 241,947 cells in the meshes for the 3-and 4-bladed turbine simulations, respectively. Fig. 7 shows the overall view of the 4-bladed turbine mesh, and the zoom-in view around the rotating sub-domains is displayed in Fig 8. The cells were concentrated near the blades, with the average wall-normal distance of $y^{+} \approx 5$. Fig. 9 shows the cells around the blades.
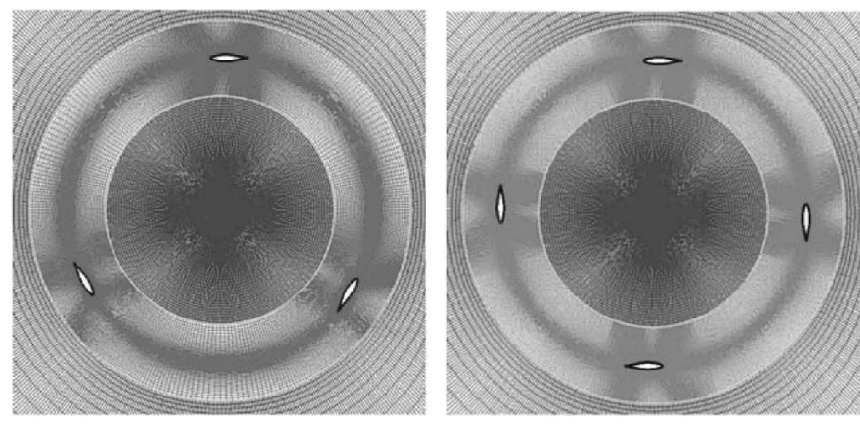

Fig. 8 Rotating sub-domain mesh for 3-and 4-bladed turbine.

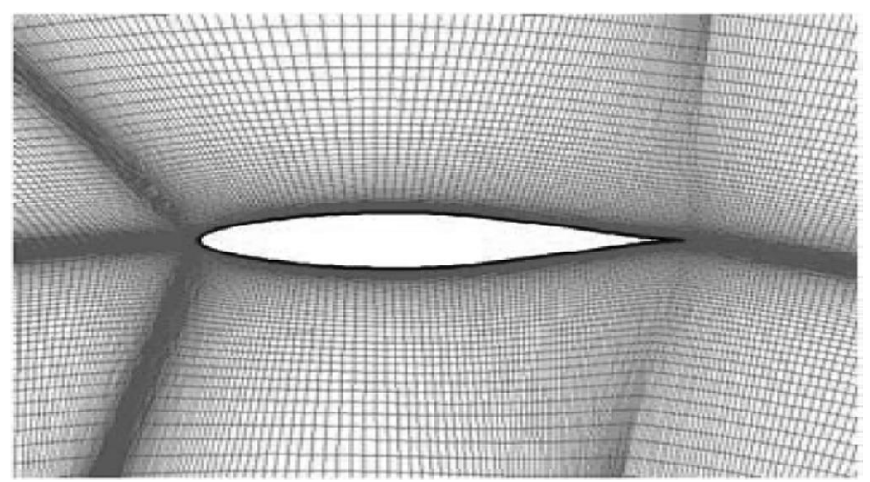

Fig. 9 Grid around the blade.

\section{RESULTS AND DISCUSSION}

A simple numerical test was performed to verify the reliability of the computational method and to determine the numerical parameters for the computation. The hydrodynamic forces acting on the $N A C A 65_{3}-018$ airfoil in different angles of attack were computed. The chord length was set to $1.524 \mathrm{~m}$, a typical size for a tidal stream turbine, and the corresponding chord-based $R e$ was 6 million. The converged solution was obtained after about 1,500 iterations. Figs. 10 to 12 show the comparison of the computational solutions and experiment data (Abbott et al., 1959; Quinn, 1944). The computed lift forces (Fig. 10) and lift-to-drag ratios (Fig. 11) are in good agreement with the data at the angles of attack between $-10^{\circ}$ and $10^{\circ}$, which is the normal operating range of the turbine blades. The difference at larger angles of attack is deemed to be due to the less accurately predicted separation and possible laminar-to-turbulent transition occurred in experiments, but not taken into account in the computation. Fig. 12 shows the surface pressure coefficient on the hydrofoil at the angle of attack of $16.5^{\circ}$. The computational solutions and experimental data are in excellent agreement.

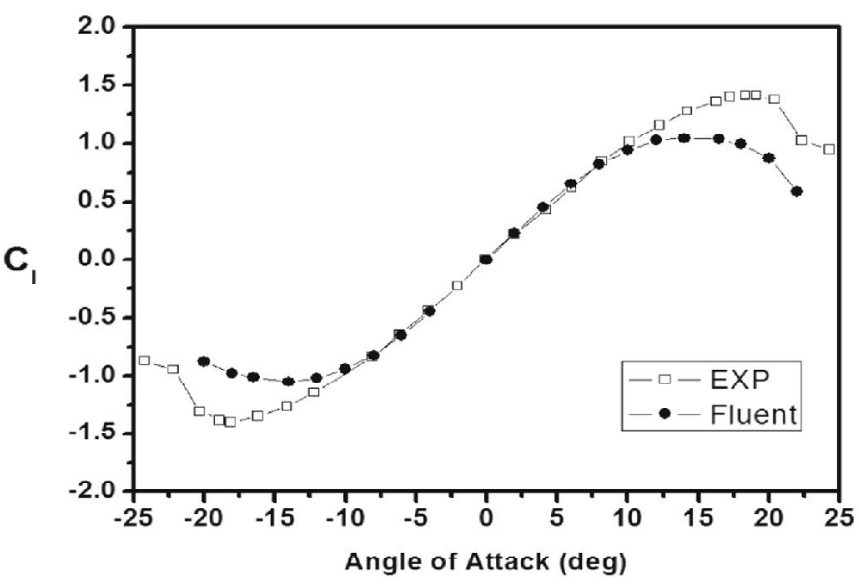

Fig. 10 Comparison of lift coefficients.

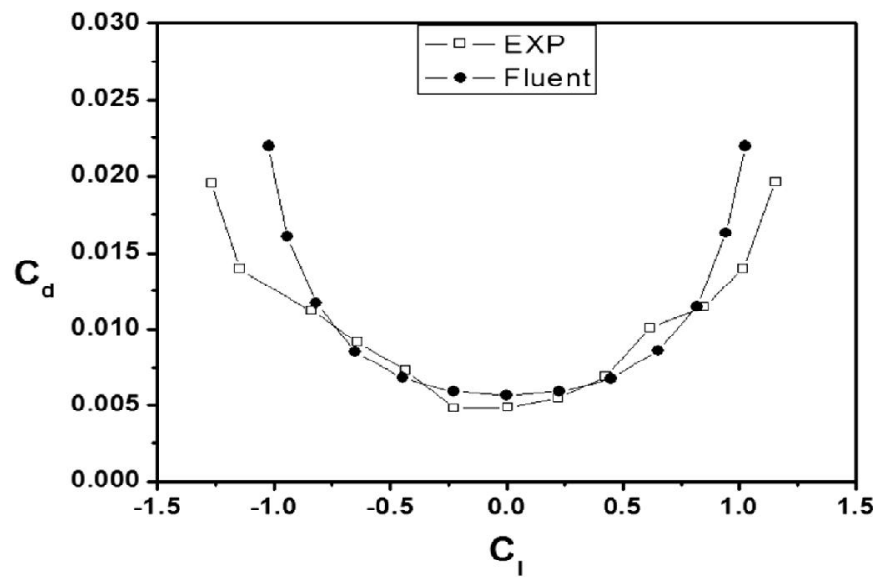

Fig. 11 Comparison of lift-to-drag ratio.

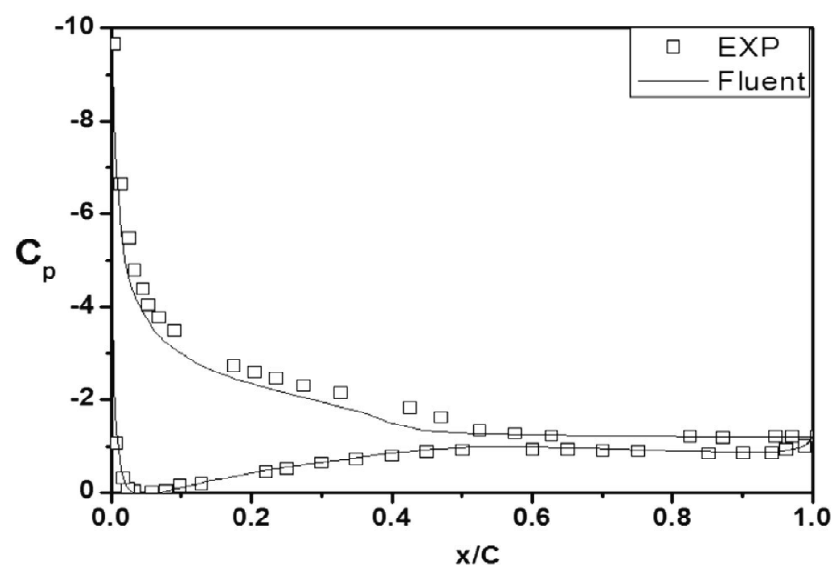

Fig. 12 Comparison of pressure distributions. 
Now with the computational method verified for the blade section geometry, the 2D unsteady flow around the turbine cross section with rotating blades was simulated. After about 2,000 iterations without the rotation of the turbine blades, the steady flow field was developed. Then, the sub-domain was let spin and the unsteady flow computation was performed. The time step size of 0.01 second was used with 80 iterations per time step. The computation was continued until the stable periodic solutions were obtained. Fig. 13 shows a snap shot of the pressure distribution. The initial position of the blades is also displayed to help understand the phase of the particular instant.
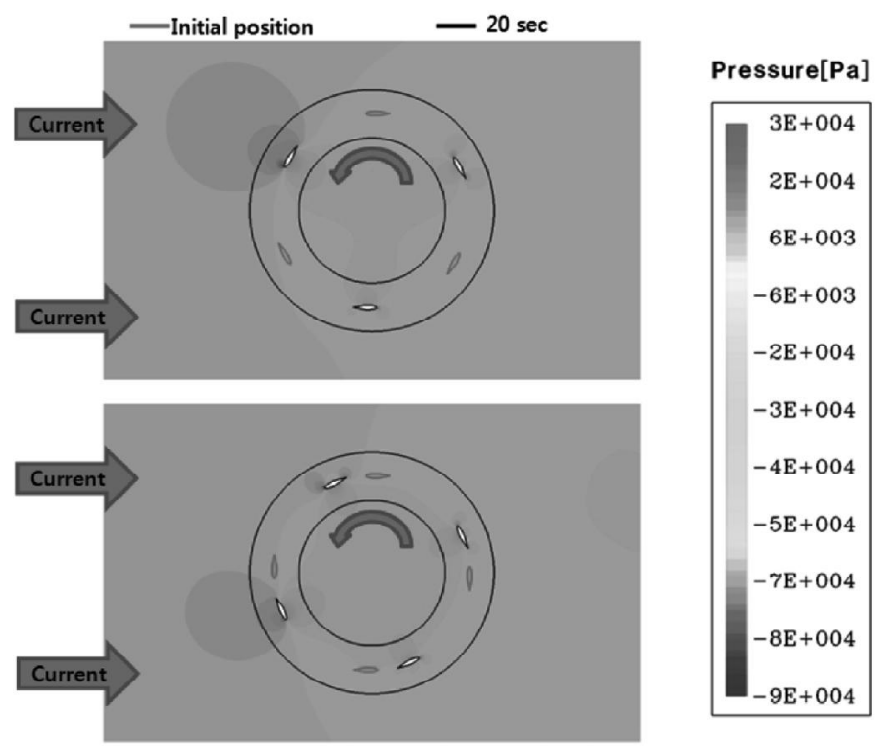

Fig. 13 Typical pressure distribution.

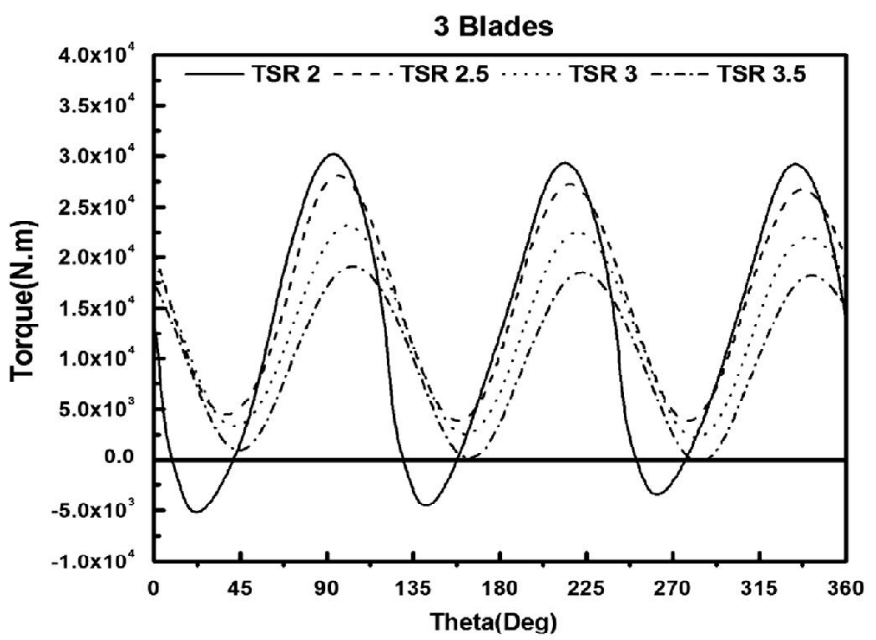

Fig. 14 Torque for different TSR (3-bladed turbine).

The sum of the torque on the blades is shown for different TSR's in Fig. 14 (3-bladed turbine) and Fig. 15 (4-bladed turbine). Three or four cycles were repeated in one rotation depending on the number of blades. As expected, with increasing number of blades, the range of torque variation was reduced. Although the maximum instant torque is larger with the 3-bladed turbine, care should be taken in calculating the average efficiency because there appears negative torque occurred at certain instants. Also note in Figs. 14 and 15 that there is an increasing phase lag with increasing number of blades. In order to maximize the turbine efficiency, the combination of the number of blades and TSR needs to be carefully considered in the initial design stage.

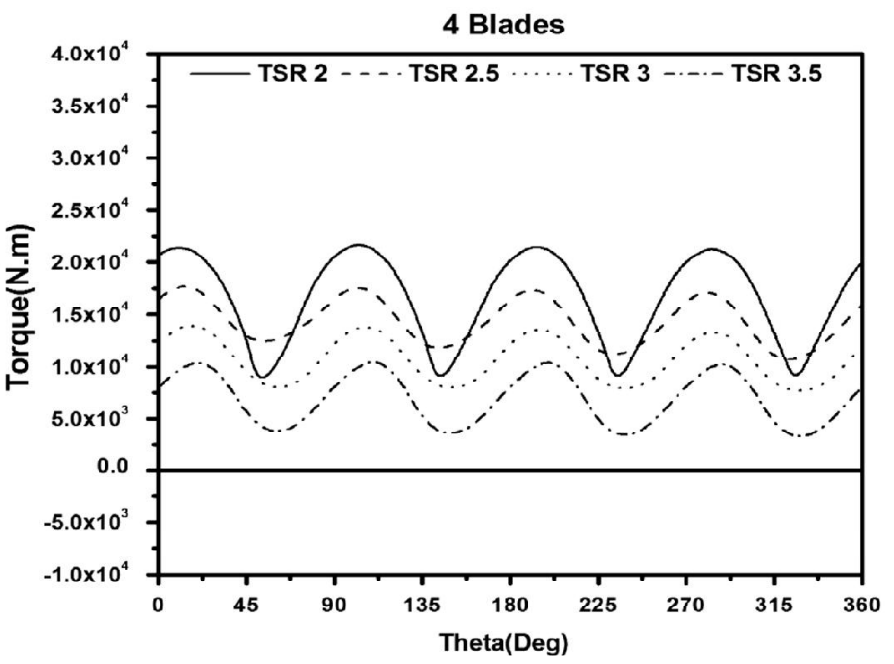

Fig. 15 Torque for different TSR (4-bladed turbine).

The shaft power is defined as Eq. (1), i.e., the time average of the torque times the angular velocity for one period. The power coefficient $\left(C_{p}\right)$ in Eq. (2) is the ratio of the average shaft power to the flow kinetic energy per unit time through the projected area of the turbine.

$P_{w}=\Omega T$

( $P_{w}:$ shaft power, $\Omega$ : angular velocity, $T$ : averaged torque)

$C_{p}=\frac{P_{w}}{\frac{1}{2} \rho V^{2}(2 R V)}=\frac{P_{w}}{\rho R V^{3}}$

The power coefficients, in other words, the efficiency of all the computed cases are shown in Fig. 16. The maximum efficiency is appeared to be obtained around TSR of 2.5 with both the 3-bladed and 4-bladed turbines. Although the overall efficiency is higher with the 3-bladed turbine, in the low TSR range, the 4-bladed turbine shows better and more stable 
performance. As mentioned above, the efficiency is influenced by both the number of blades and TSR, and thereby these variables must be selected with special care.

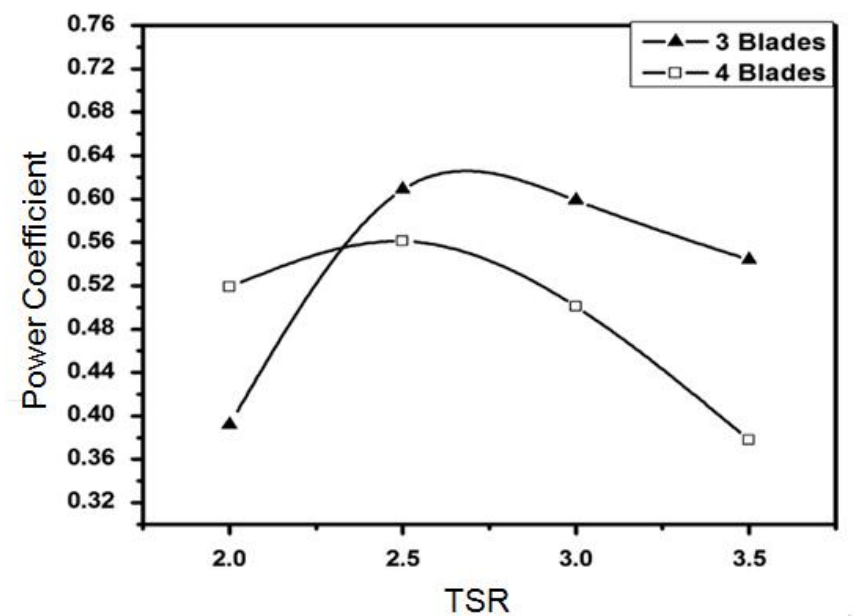

Fig. 16 Power coefficients vs. TSR for 3- and 4-bladed turbines.

\section{CONCLUSIONS}

The 2D unsteady flow analysis for a VAT cross section with fixed-pitch three or four blades was performed using an unsteady RANS method. Firstly, the hydrodynamic forces acting on the $N A C A 65_{3}-018$ airfoil in different angles of attack were computed and the results were compared with experimental data. By employing the sliding mesh method, the unsteady flow field around a VAT with rotating blades was simulated. It is confirmed that a proper combination of the number of blades and TSR is essential for the optimum efficiency of the turbine. The maximum efficiency of the simplified 2D model was obtained at TSR of 2.5. The effects of pitch angle and blade skew are planned to be investigated as future work.

\section{ACKNOWLEDGMENTS}

This study was sponsored by the Ministry of Knowledge Economy through Projects No. 2009T100100245 and No. 2009T100100556).

\section{REFERENCES}

Abbott, I.H. Von Doenhoff, A.E. and Stivers, Jr, L.S., 1959. Summary of airfoil data. NACA Report 824.

Glauert, H., 1935. Airplane propellers. In: Durand, W. F., ed. 1963. Aerodynamic Theory. III \& IV, Division L, New York: Dover Publication Inc.

Menter, F.R., 1993. Zonal two equation turbulence models for aerodynamics flows. AIAA Paper 93-2906.

Ockfen, A.E. and Matveev, K.I., 2009. Aerodynamic characteristics of NACA 4412 airfoil section with flap. Journal of Naval Architecture and Ocean Engineering, 1(1), pp.1-12.

Patankar, S.V. and Spalding, D.B., 1972. A calculation procedure for heat, mass and momentum transfer in three-dimensional parabolic flows. International Journal of Heat and Mass Transfer, 15, pp.1787-1806.

Quinn, J.H. Jr, 1944. Tests of the NACA airfoil section with boundary-layer control by suction. NACA wartime reports L209.

Rhee, S.H., 2009. RANS method for free-surface wave flows around surface-piercing cylindrical structures. $A S C E$ Journal of Waterway, Port, Coastal, and Ocean Engineering, 135(4), pp.135-143.

Rhee, S.H. and Kim, H., 2008. A suggestion of gap flow control devices for the suppression of rudder cavitation. Journal of Marine Science and Technology, 13(4), pp.356-370.

Sorensen, N.N. Michelsen, J.A. and Schreck, S., 2002. Navier-Stokes predictions of the NREL Phase VI Rotor in the NASA Ames $80 \mathrm{ft} \times 120 \mathrm{ft}$ wind tunnel. Wind Energy, 5, pp.151-169. 